\title{
Normative data for multiple breath washout outcomes in school-aged Caucasian children
}

\author{
Pinelopi Anagnostopoulou (1012,3, Philipp Latzin (1) ${ }^{1}$, Renee Jensen ${ }^{4}$, \\ Mirjam Stahl (10,6, Alana Harper ${ }^{7}$, Sophie Yammine ${ }^{1}$, Jakob Usemann (1) ${ }^{1,8}$, \\ Rachel E. Foong ${ }^{7,9}$, Ben Spycher ${ }^{10}$, Graham L. Hall ${ }^{7,9}$, Florian Singer (1) ${ }^{1}$, \\ Sanja Stanojevic ${ }^{4}$, Marcus A. Mall $\mathbb{1}^{5,6,11,12}$, Felix Ratjen ${ }^{4,13}$ and \\ Kathryn A. Ramsey ${ }^{1,7,13}$
}

@ERSpublications

This study provides reference values for nitrogen multiple breath washout outcomes in healthy Caucasian children from 6 to 18 years old, measured with a commercially available device http://bit.ly/ 2rsab8v

Cite this article as: Anagnostopoulou P, Latzin P, Jensen R, et al. Normative data for multiple breath washout outcomes in school-aged Caucasian children. Eur Respir J 2020; 55: 1901302 [https://doi.org/ 10.1183/13993003.01302-2019].

\section{ABSTRACT}

Background: The multiple breath nitrogen washout $\left(\mathrm{N}_{2} \mathrm{MBW}\right)$ technique is increasingly used to assess the degree of ventilation inhomogeneity in school-aged children with lung disease. However, reference values for healthy children are currently not available. The aim of this study was to generate reference values for $\mathrm{N}_{2} \mathrm{MBW}$ outcomes in a cohort of healthy Caucasian school-aged children.

Methods: $\mathrm{N}_{2} \mathrm{MBW}$ data from healthy Caucasian school-age children between 6 and 18 years old were collected from four experienced centres. Measurements were performed using an ultrasonic flowmeter (Exhalyzer D, Eco Medics AG, Duernten, Switzerland) and were analysed with commercial software (Spiroware version 3.2.1, Eco Medics AG). Normative values and upper limits of normal (ULN) were generated for lung clearance index (LCI) at 2.5\% $\left(\mathrm{LCI}_{2.5 \%}\right)$ and at $5 \%\left(\mathrm{LCI}_{5 \%}\right)$ of the initial nitrogen concentration and for moment ratios $\left(M_{1} / M_{0}\right.$ and $\left.M_{2} / M_{0}\right)$. A prediction equation was generated for functional residual capacity (FRC).

Results: Analysis used 485 trials from 180 healthy Caucasian children aged from 6 to 18 years old. While LCI increased with age, this increase was negligible $\left(0.04\right.$ units $y e a r^{-1}$ for $\left.\mathrm{LCI}_{2.5 \%}\right)$ and therefore fixed ULN were defined for this age group. These limits were 7.91 for $\mathrm{LCI}_{2.5 \%}, 5.73$ for $\mathrm{LCI}_{5 \%}, 1.75$ for $\mathrm{M}_{1} / \mathrm{M}_{0}$ and 6.15 for $\mathrm{M}_{2} / \mathrm{M}_{0}$, respectively. Height and weight were found to be independent predictors of FRC.

Conclusion: We report reference values for $\mathrm{N}_{2} \mathrm{MBW}$ outcomes measured on a commercially available ultrasonic flowmeter device (Exhalyzer D, Eco Medics AG) in healthy school-aged children to allow accurate interpretation of ventilation distribution outcomes and FRC in children with lung disease.

After publication of this study, an error in the cross-sensitivity correction of the oxygen and carbon dioxide sensors in the Exhalyzer D device has been identified that leads to an overestimation of multiple breath washout outcomes. Please refer to the correction notice published in the August 2022 issue of the European Respiratory Journal for further details.

This article has an editorial commentary: https://doi.org/10.1183/13993003.00485-2020.

This article has supplementary material available from erj.ersjournals.com

Received: 2 July 2019 | Accepted after revision: 10 Dec 2019

Copyright OERS 2020 


\section{Introduction}

The multiple breath washout (MBW) test provides lung volume and ventilation distribution outcomes that are more sensitive than conventional lung function outcomes for detecting lung disease in children with cystic fibrosis (CF) [1-3] and potentially other respiratory disorders $[4,5]$. The lung clearance index (LCI) is a global marker of ventilation distribution derived from the MBW test that is reproducible [6, 7], discriminates between health and disease [3], and correlates with the extent of structural lung disease [2, 8] in children with CF. These data have led to LCI being used as the primary endpoint in observational studies and interventional trials in patients with $\mathrm{CF}$ [9-11]. It may potentially also be a future factor for the clinical surveillance of children with CF [12].

LCI is traditionally calculated as the number of lung turnovers required to wash out a tracer gas to one fortieth (1/40th) of its starting concentration $\left(\mathrm{LCI}_{2.5 \%}\right)$ [13]. The number of lung turnovers required to wash out a tracer gas to one twentieth $(1 / 20 \mathrm{th})$ of its initial concentration $\left(\mathrm{LCI}_{5 \%}\right)$ has also been calculated $[14,15]$. Moment ratios $\left(\mathrm{M}_{1} / \mathrm{M}_{0}\right.$ and $\left.\mathrm{M}_{2} / \mathrm{M}_{0}\right)$ describe the degree of skewness of the washout curve and may be more sensitive for detecting ventilation inhomogeneity in the periphery of the lung $[2,15]$. In addition, the MBW test measures the functional residual capacity (FRC) of the lung, which may indicate some degree of lung hyperinflation [1].

Despite the increasing use of MBW, reference values for MBW outcomes in children are scarce [16, 17]. Several commercially available and custom-made MBW devices are currently in use but outcomes are generally not interchangeable between them [18-20]. In addition, different software versions and system settings can influence the calculation of MBW outcomes [21, 22]. As a result, published normative values are only applicable for the specific device, software and tracer gas used [17, 21, 23]. For this reason, studies evaluating MBW outcomes using commercially available equipment have required data collection in age-matched healthy controls $[2,12]$.

The aim of our study was to provide normative values for multiple breath nitrogen washout $\left(\mathrm{N}_{2} \mathrm{MBW}\right)$ outcomes (LCI, moment ratios and FRC) in school-aged children, as measured with a commercially available device (the Exhalyzer D ultrasonic flowmeter (Eco Medics AG, Duernten, Switzerland)) and to investigate the association of $\mathrm{MBW}$ outcomes with anthropometric and physiological parameters, including tidal volume $\left(V_{\mathrm{T}}\right)$ and equipment-related dead space volume $\left(V_{\mathrm{D}}\right)$ [24, 25]. To achieve this, we collected data from healthy children in four different centres using the same MBW device and protocol, and analysed these data using the same software and system settings.

\section{Methods}

\section{Study subjects}

For this study we used MBW measurements from healthy school-aged Caucasian children collected between 2011 and 2016 from four paediatric centres specialised in $\mathrm{N}_{2} \mathrm{MBW}$, including Inselspital (Bern, Switzerland), SickKids (Toronto, Canada), University Children's Hospital (Heidelberg, Germany) and Telethon Kids Institute (Perth, Australia). We used the following exclusion criteria: non-Caucasians, age less than 6 years or greater than 18 years, chronic respiratory or cardiac disease, respiratory infection during the last 4 weeks prior to measurement and other major systemic diseases with potential influence on lung function [26]. Healthy individuals from each centre took part in prospective observational studies and therefore some data have been published previously $[2,8,12,18,27,28]$. The study was approved by local ethics committees (Ethics Committee of the Canton of Bern, Bern, Switzerland; Research Ethics Board at SickKids, Toronto, Canada; Ethics Committee of the University of Heidelberg, Heidelberg, Germany; Princess Margaret Hospital Human Ethics Committee, Perth, Australia). Parents or caregivers provided informed written consent.

Affiliations: 'Division of Respiratory Medicine, Dept of Pediatrics, Inselspital, University of Bern, Bern, Switzerland. ${ }^{2}$ Insitute of Anatomy, University of Bern, Bern, Switzerland. ${ }^{3}$ Medical School, University of Cyprus, Nicosia, Cyprus (current affiliation). ${ }^{4}$ Division of Respiratory Medicine, The Hospital for Sick Children and Translational Medicine, SickKids Research Institute, University of Toronto, Toronto, ON, Canada. ${ }^{5}$ Dept of Translational Pulmonology, Translational Lung Research Center Heidelberg (TLRC), German Center for Lung Research (DZL), University of Heidelberg, Heidelberg, Germany. 'Division of Pediatric Pulmonology and Allergy and Cystic Fibrosis Center, Dept of Pediatrics, University of Heidelberg. Heidelberg. Germany. ${ }^{7}$ Telethon Kids Institute, Perth, Australia. ${ }^{8}$ University of Basel Children's Hospital (UKBB), Basel, Switzerland. ${ }^{9}$ School of Physiotherapy and Exercise Science, Curtin University, Perth, Australia. ${ }^{10}$ Institute of Social and Preventive Medicine, University of Bern, Bern, Switzerland. ${ }^{11}$ Dept of Pediatric Pulmonology, Immunology and Intensive Care Medicine, Charité-Universitätsmedizin Berlin, Berlin, Germany. ${ }^{12}$ Berlin Institute of Health (BIH), Berlin, Germany. ${ }^{13} \mathrm{~F}$. Ratjen and K.A. Ramsey contributed equally as last authors.

Correspondence: Felix Ratjen, Research Institute, The Hospital for Sick Children, Division of Respiratory Medicine, Department of Pediatrics, 555 University Avenue, ON M5G 1X8, Toronto, Canada. E-mail: felix.ratjen囚 sickkids.ca 


\section{MBW measurements}

We performed $\mathrm{N}_{2} \mathrm{MBW}$ measurements using an ultrasonic flowmeter (Exhalyzer D, Eco Medics AG, Duernten, Switzerland) and the software provided by the manufacturer (Spiroware version 3.1.6, Eco Medics AG) as previously described $[12,18]$. All centres used the same equipment and performed the calibration and measurement using the same protocol (details are available in the supplementary material).

\section{MBW analysis}

$\mathrm{N}_{2} \mathrm{MBW}$ data originally recorded in Spiroware version 3.1.6 were reloaded and analysed using the updated version of the software provided by the manufacturer at the time the study was performed (Spiroware version 3.2.1, Eco Medics AG). Further details are provided in the supplementary material.

\section{MBW outcomes and physiological indices}

FRC, $\mathrm{LCI}_{2.5 \%}, \mathrm{LCI}_{5 \%}$ and moment ratios $\left(\mathrm{M}_{1} / \mathrm{M}_{0}\right.$ and $\left.\mathrm{M}_{2} / \mathrm{M}_{0}\right)$ were calculated according to current recommendations $[13,14]$. The mean $V_{\mathrm{T}}$ for each trial was provided by the software. In order to investigate the effect of breathing pattern [29] and equipment-related $V_{\mathrm{D}}$ [30] on MBW outcomes, the mean ratio of $V_{\mathrm{T}}$ to $\operatorname{FRC}\left(V_{\mathrm{T}} / \mathrm{FRC}\right)$ and $V_{\mathrm{D}}$ to $V_{\mathrm{T}}\left(V_{\mathrm{D}} / V_{\mathrm{T}}\right)$ were calculated per trial (expressed as percentages).

\section{Quality control of MBW trials}

Quality control of MBW trials was assessed by an experienced operator at the Inselspital, Bern (P. Anagnostopoulou) according to the 2013 American Thoracic Society (ATS)/European Respiratory Society (ERS) MBW consensus guidelines [13] and additional criteria proposed by JENSEN et al. [31]. Further details are provided in the supplementary material. Tests with at least two technically acceptable trials with FRC values within $25 \%$ of the mean were included for analysis.

\section{Statistics}

Statistical analysis of the data was performed using $\mathrm{R}$ version 3.4.3 (The $\mathrm{R}$ Project for Statistical Computing, www.r-project.org) and Stata statistical software, release 14 (StataCorp LP, College Station, TX, USA). Graphs were generated using GraphPad Prism version 5.0 (GraphPad Software, San Diego, CA, USA). Normality of data distribution was assessed visually and using the Shapiro-Wilk test. The upper and lower limits of normal (ULN and LLN, respectively) correspond to the 97.5th percentile (mean $\pm 1.96 \mathrm{SD})$, as previously described [17]. The intra-subject variability was defined as the mean relative difference for subjects with two trials $\left(\left(\operatorname{trial}_{1}-\operatorname{trial}_{2}\right) /\right.$ mean $\left.(\%)\right)$ or the coefficient of variation $(\mathrm{CV}$; sD/mean (\%)) for those with at least three trials. We used one-way ANOVA with a Bonferroni post-hoc test for between-centre comparisons. Multiple linear regression was performed to assess the associations of normally distributed primary outcomes ( $\mathrm{LCI}_{2.5 \%}, \mathrm{LCI}_{5 \%}, \mathrm{M}_{1} / \mathrm{M}_{0}, \mathrm{M}_{2} / \mathrm{M}_{0}$ and $\mathrm{FRC}$ ) with demographic factors (age, weight, height and sex) and physiological factors $\left(V_{\mathrm{T}} / \mathrm{FRC}\right.$ and $\left.V_{\mathrm{D}} / V_{\mathrm{T}}\right)$. All of the variables considered were selected for biological reasons. Multicollinearity between independent variables was assessed by checking the variance inflation factor and was not present in the final models. The homoscedasticity and normality of residuals for the models were assessed using White's test and the Shapiro-Wilk test, respectively. In the final models, only significant factors were considered to facilitate easy application of the reference equations. The statistical significance level was set to $\mathrm{p}<0.05$.

\section{Results}

\section{Study subjects}

$\mathrm{N}_{2} \mathrm{MBW}$ data from 285 healthy children were assessed for eligibility. Of these, 67 did not fulfil the inclusion criteria and 38 did not pass the quality control (success rate: $82.6 \%$ ) (figure 1). Thus, $485 \mathrm{MBW}$ trials from 180 children were used for analysis: 82 children from the University Children's Hospital in Bern, Switzerland (mean age: 10.8 years; range: 6.0-17.8 years), 33 children from the University Children's Hospital Heidelberg, Germany (mean age: 12.0 years; range: $7.1-17.0$ years), 28 children from the SickKids in Toronto, Canada (mean age: 12.5 years; range: 6.5-17.2 years) and 37 children from the Telethon Kids Institute, Perth, Australia (mean age: 9.5 years; range: $6.0-13.6$ years). Of the 180 study participants, $38.9 \%$ had two acceptable MBW trials, $53.9 \%$ had three acceptable trials and $7.2 \%$ had four or more acceptable trials.

\section{Centre differences}

Study demographics and anthropometrics for each centre are reported in table 1. Participants from Perth were significantly younger compared with those from Toronto and Heidelberg $(p<0.001)$ (supplementary figure S1). Participants from Toronto had higher height $\mathrm{z}$-scores compared with those from Heidelberg ( $p=0.02$ ) but there were no differences in weight $\mathrm{z}$-score between centres. There were no differences in LCI or moment ratio outcomes between centres; however, there were significant differences in FRC, whereby 
FIGURE 1 Flow chart of healthy subjects who participated in the study

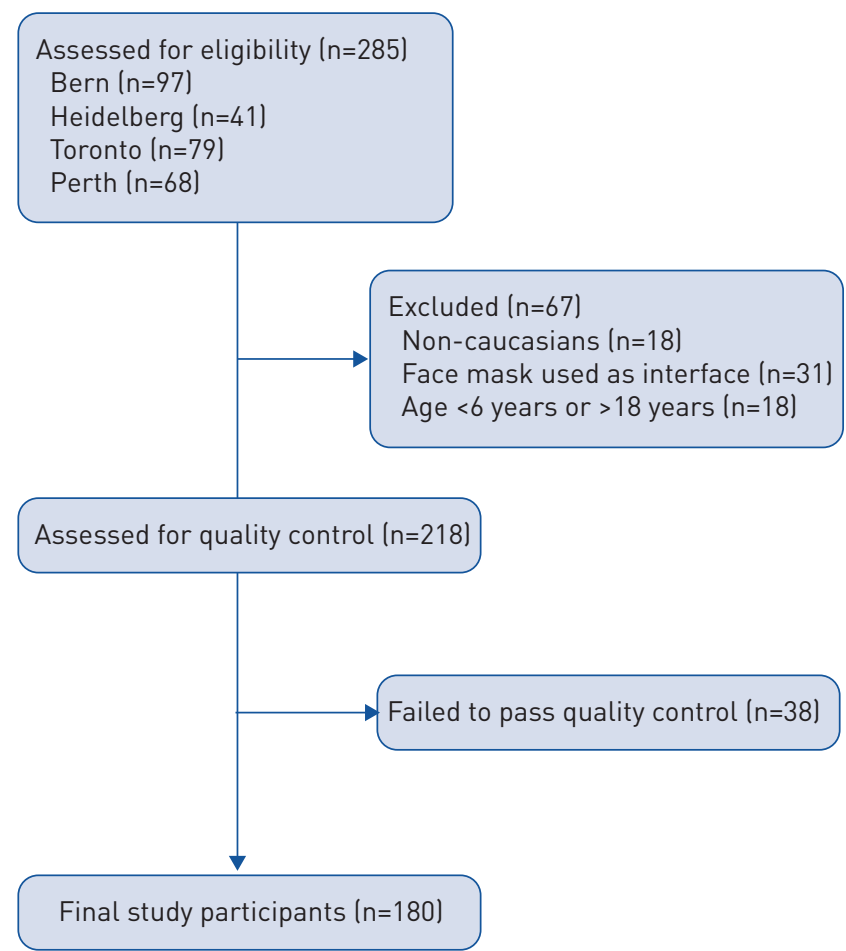

children from Toronto had higher FRC values compared to children from Perth ( $p=0.02)$ (supplementary figure S1).

\section{Lung clearance index and moment ratios}

In univariable regression analyses, $\mathrm{LCI}_{2.5 \%}$ was negatively associated with height (coefficient $-0.005,95 \%$ $\mathrm{CI}-0.0087$ to $-0.0019 ; \mathrm{p}=0.002$ ), weight (coefficient $-0.0049,95 \% \mathrm{CI}-0.0090$ to $-0.0008 ; \mathrm{p}=0.020$ ) and age (coefficient $-0.0225,95 \% \mathrm{CI}-0.0421$ to $-0.0030 ; \mathrm{p}=0.024$ ), and positively associated with $V_{\mathrm{D}} / V_{\mathrm{T}}$ (coefficient 0.0258 , 95\% CI $0.0093-0.0422 ; \mathrm{p}=0.002$ ). No association was found between $\mathrm{LCI}_{2.5 \%}$ and sex or $V_{\mathrm{T}} / \mathrm{FRC}(\mathrm{p}>0.05)$.

Similarly, $\mathrm{LCI}_{5 \%}$ and moment ratio outcomes were negatively associated with height, weight and age (supplementary table S1). In addition, $V_{\mathrm{D}} / V_{\mathrm{T}}$ and $V_{\mathrm{T}} / \mathrm{FRC}$ were both negatively associated with age

\section{TABLE 1 Demographic and anthropometric characteristics of the study population per centre}

\begin{tabular}{|c|c|c|c|c|c|}
\hline & Bern & Toronto & Heidelberg & Perth & Total \\
\hline Subjects n & 82 & 28 & 33 & 37 & 180 \\
\hline Male sex $n$ & 38 & 19 & 20 & 15 & 92 \\
\hline Trials n & 224 & 98 & 86 & 84 & 492 \\
\hline Age years & $10.8 \pm 3.8$ & $12.5 \pm 3.1$ & $12.0 \pm 2.6$ & $9.5 \pm 2.0$ & $11.0 \pm 3.3$ \\
\hline \multicolumn{6}{|l|}{ Weight $^{\#}$} \\
\hline In kg & $39.8 \pm 17.0$ & $47.5 \pm 15.5$ & $43.1 \pm 16.0$ & $34.2 \pm 10.4$ & $40.5 \pm 15.9$ \\
\hline By z-score & $0.21 \pm 0.83$ & $0.34 \pm 0.93$ & $0.01 \pm 1.02$ & $0.27 \pm 0.77$ & $0.21 \pm 0.87$ \\
\hline \multicolumn{6}{|l|}{ Height $^{\#}$} \\
\hline In $\mathrm{cm}$ & $144.8 \pm 21.9$ & $155.9 \pm 17.8$ & $148.5 \pm 14.6$ & $139.1 \pm 12.8$ & $146.0 \pm 19.1$ \\
\hline By z-score & $0.40 \pm 1.00$ & $0.69 \pm 1.40$ & $-0.10 \pm 1.06$ & $0.51 \pm 0.69$ & $0.38 \pm 1.05$ \\
\hline \multicolumn{6}{|l|}{ BMI $^{\#}$} \\
\hline $\mathrm{ln} \mathrm{kg} \cdot \mathrm{m}^{-2}$ & $18.0 \pm 3.2$ & $19.0 \pm 3.6$ & $19.0 \pm 2.8$ & $17.3 \pm 2.7$ & $18.2 \pm 3.1$ \\
\hline By z-score & $0.09 \pm 0.90$ & $0.19 \pm 1.03$ & $0.25 \pm 1.20$ & $0.04 \pm 0.83$ & $0.12 \pm 0.97$ \\
\hline FRC L & $1.77 \pm 0.89$ & $2.19 \pm 0.87$ & $1.78 \pm 0.62$ & $1.50 \pm 0.38$ & $1.78 \pm 0.78$ \\
\hline
\end{tabular}

Data are presented as mean \pm SD unless otherwise stated. BMI: body mass index; FRC: functional residual capacity; CDC: Centers for Disease Control and Prevention. "\#: weight, height and BMI z-scores were calculated according to CDC growth charts [32]. 
(supplementary figure S2), while $\mathrm{LCI}_{5 \%}$ and $\mathrm{M}_{1} / \mathrm{M}_{0}$ were positively associated with $V_{\mathrm{D}} / V_{\mathrm{T}}$ and $V_{\mathrm{T}} / \mathrm{FRC}$ (supplementary table S1 and supplementary figure S2). There was no evidence for association of $\mathrm{LCI}_{5 \%}$ and moment ratios with sex ( $\mathrm{p}>0.05)$ (supplementary table $\mathrm{S} 1)$.

In a multivariable regression model age, $V_{\mathrm{D}} / V_{\mathrm{T}}$ and $V_{\mathrm{T}} / \mathrm{FRC}$ were independently associated with LCI and moment ratio outcomes (supplementary table S2). However, the regression coefficients for each of these independent predictors were small $(\mathrm{r} \leqslant 0.10)$. For $\mathrm{LCI}_{2.5 \%}$ the coefficient for age was 0.04 , indicating that, after adjusting for $V_{\mathrm{D}} / V_{\mathrm{T}}$ and $V_{\mathrm{T}} / \mathrm{FRC}, \mathrm{LCI}_{2.5 \%}$ would increase by 0.04 units.year ${ }^{-1}$ (95\% CI 0.01-0.07). This equates to a maximum 0.5 unit change in $\mathrm{LCI}_{2.5 \%}$ over 12 years (from 6 years to 18 years of age). Thus, in our judgement, the age related changes in LCI and moment ratios are negligible. Therefore, we report fixed ULN during this age interval (figure 2).

The mean LLN and ULN for LCI and moment ratios are provided in table 2. The ULN for LCI $_{2.5 \%}$ was 7.91 and for $\mathrm{LCI}_{5 \%}$ was 5.73. For $\mathrm{M}_{1} / \mathrm{M}_{0}$ the ULN was 1.75 and for $\mathrm{M}_{2} / \mathrm{M}_{0}$ was 6.15 (figure 2).

\section{Functional residual capacity}

FRC values were right skewed and therefore natural $\log (\ln )$ transformed FRC values and predictors were used in the models. $\operatorname{Ln}\left(\right.$ FRC) was positively associated with $\ln$ (height), $\ln$ (weight), $\ln \left(V_{\mathrm{T}}\right)$ and $\ln ($ age $)$, and negatively associated with $\ln \left(V_{\mathrm{D}} / V_{\mathrm{T}}\right)$ in the univariate analysis (figure 3 and supplementary table 1 ). There was no evidence of association with sex $(\mathrm{p}=0.68)$ (supplementary table 1$)$. In a multivariable model, $\ln$ (FRC) was independently associated with $\ln$ (height) and $\ln$ (weight). There were no differences in FRC $\mathrm{z}$-scores between centres $(\mathrm{p}=0.81)$. The full FRC prediction equation, including the intercept for the model, is provided in the supplementary material. See also equations 1 and 2 (where FRC is expressed in $\mathrm{L}$, height in $\mathrm{cm}$ and weight in $\mathrm{kg}$ ).

$$
\begin{gathered}
\text { Predicted FRC }=e^{-18.18} \cdot \text { height }^{3.98} \cdot \text { weight }^{-0.32} \\
\text { z-score FRC }=\frac{\ln (\text { measured FRC }- \text { predicted FRC })}{0.1632}
\end{gathered}
$$
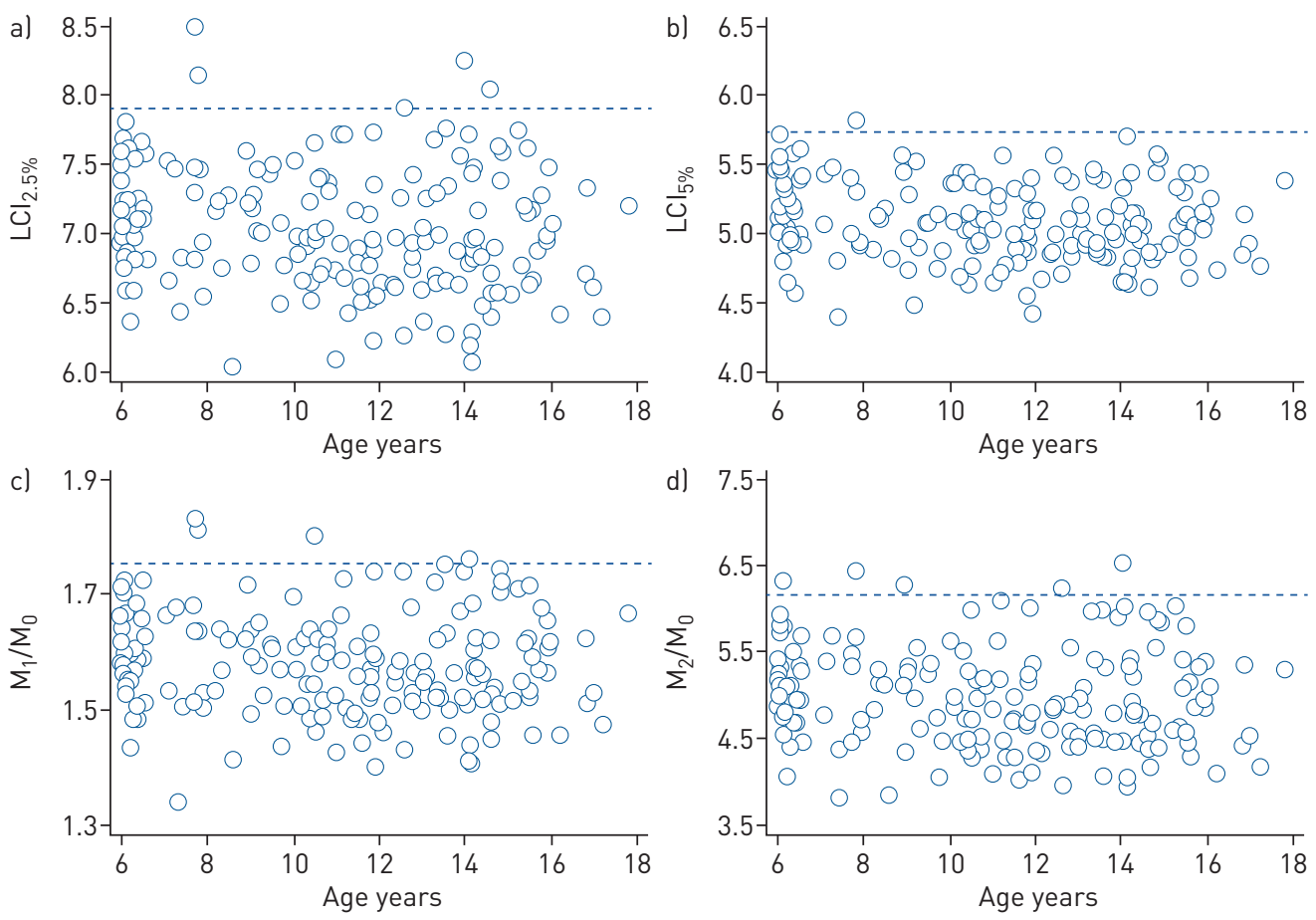

FIGURE 2 Relationship between age and lung clearance index (LCl) at (a) $2.5 \%\left(\mathrm{LCl}_{2.5 \%}\right)$, (b) $5 \%\left(\mathrm{LCl}_{5} \%\right.$ ), (c) moment ratio one $\left(M_{1} / M_{0}\right)$ and (d) moment ratio two $\left(M_{2} / M_{0}\right)$. Dashed lines indicate the upper limit of normal (ULN) 
TABLE 2 Variability in multiple breath nitrogen washout $\left(\mathrm{N}_{2} \mathrm{MBW}\right)$ outcomes from 180 healthy children

\begin{tabular}{lcccc} 
Outcome & Mean \pm SD & LLN $^{\#}$ & ULN $^{\#}$ & Within-test intra-subject variability \% $\$$ SD \\
\hline $\mathbf{L C l}_{\mathbf{2 . 5} \%}$ & $7.04 \pm 0.45$ & 6.16 & 7.91 & $4.77 \pm 3.16$ \\
$\mathbf{L C l}_{\mathbf{5} \%}$ & $5.10 \pm 0.32$ & 4.47 & 5.73 & $4.47 \pm 2.89$ \\
$\mathbf{M}_{\mathbf{1}} / \mathbf{M}_{\mathbf{0}}$ & $1.58 \pm 0.09$ & 1.40 & 1.75 & $4.10 \pm 2.67$ \\
$\mathbf{M}_{\mathbf{2}} / \mathbf{M}_{\mathbf{0}}$ & $4.97 \pm 0.60$ & 3.79 & 6.15 & $8.07 \pm 5.22$
\end{tabular}

Data are presented as mean \pm SD unless otherwise stated. LLN: lower limit of normal; ULN: upper limit of normal; $\mathrm{LCl}_{\mathrm{x}}$ : lung clearance index at $\mathrm{x} \%$ of the initial nitrogen concentration; $\mathrm{M}_{1} / \mathrm{M}_{0}$ : moment ratio one; $\mathrm{M}_{2} / \mathrm{M}_{0}$ : moment ratio two. " : LLN and ULN were calculated as mean $\pm 1.96 \mathrm{sD}$. The intra-subject variability was calculated as the \% difference $\left(\left(\right.\right.$ trial $\left._{1}-\operatorname{trial}_{2}\right) /$ mean $\left.(\%)\right)$ for subjects with two trials and as the coefficient of variation (CV; sD/mean (\%)) for subjects with three or more trials.

\section{Discussion}

Normative $\mathrm{N}_{2} \mathrm{MBW}$ data for a paediatric Caucasian population between the ages of 6 and 18 years is reported using commercially available equipment and software. While a significant age dependency is observed for LCI and moment ratio outcomes, the magnitude of this effect is small during the age interval of 12 years. Therefore, fixed ULN for LCI and moment ratios can be used in this age group. FRC was independently predicted by both height and weight, and FRC predicted values and a $\mathrm{z}$-score equation are provided.

\section{Comparison with the literature}

The $\mathrm{LCI}_{2.5 \%}$ values in our cohort are slightly higher than previously reported for this age group $[16,17,24]$. However, it is difficult to perform a direct comparison with previous studies due to differences in equipment and software algorithms. Lum et al. [17] reported an ULN for $\mathrm{LCI}_{2.5 \%}$ of 7.53 in healthy children who performed $\mathrm{MBW}$ measurements using a mass spectrometer with sulfur hexafluoride $\left(\mathrm{SF}_{6}\right)$ as the tracer gas. However, several studies have reported higher $\mathrm{LCI}_{2.5 \%}$ values in $\mathrm{N}_{2} \mathrm{MBW}$ compared with $\mathrm{SF}_{6} \mathrm{MBW}[18,33$, 34]. These differences may be explained by different distribution of a resident gas compared with an exogenous gas in the lung tissue and/or by the contribution of nitrogen diffusion from the lung tissue during the washout $[18,20]$. FuCHs et al. [16] reported an ULN for $\operatorname{LCI}_{2.5 \%}$ of 7.0 in healthy children who performed $\mathrm{SF}_{6} \mathrm{MBW}$ using a device which utilises a similar ultrasonic flowmeter measurement principle. Recent data show that this device provides lower $\mathrm{LCI}_{2.5 \%}$ values compared to the device used in our study $[19,35]$. In addition, HoulTz et al. [24] reported an ULN for $\mathrm{LCI}_{2.5 \%}$ of 7.09 in healthy children who performed $\mathrm{N}_{2} \mathrm{MBW}$ using the same equipment used in our study; however, they analysed their data using custom-made software and as such a direct comparison between the two datasets is not possible. These findings highlight the need for equipment and software specific normative data for MBW data.

\section{Centre differences}

Slight differences in height and weight between centres were not surprising because the age distribution of the study participants differed between these centres. Previous multi-centre studies have reported differences in MBW indices using the same measuring equipment and protocol $[17,36]$. In order to
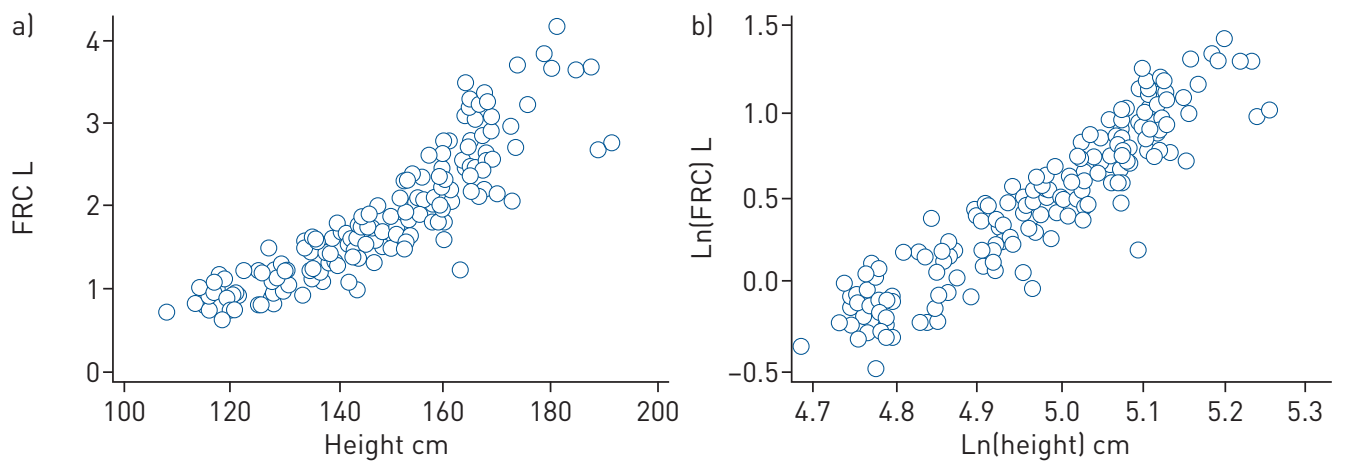

FIGURE 3 Relationship between functional residual capacity (FRC) and height expressed (a) linearly or (b) as natural logarithms 
minimise the risk for between-centre differences, the analysis was performed using the same software version, system settings and equipment-related $V_{\mathrm{D}}$. We did not find any differences in LCI and moment ratio outcomes between centres. Small but statistically significant differences were found in FRC between centres; however, FRC z-scores were not different between centres. Therefore we hypothesise that any differences in FRC between centres were simply due to differences in demographic data.

\section{Predictors of MBW outcomes}

Previous studies have reported that $\mathrm{LCI}_{2.5 \%}$ is age dependent from birth to adulthood [17, 25]; however, it is unclear whether this age dependence continues during school age and adolescence. Lum et al. [17] reported that despite a small continuation of decline in $\mathrm{LCI}_{2.5 \%}$ throughout the entire paediatric range, changes were minimal once the child reached 6 years of age. The authors reported that fixed ULN could be used for $\mathrm{LCI}_{2.5 \%}$ between the ages of 6 and 19 years. We report small but statistically significant associations between age and LCI as well as between age and moment ratios. However, the changes in LCI and moment ratios with age during the school age period are minimal, as shown in figure 2 . Therefore we believe that ULN for LCI and moment ratios are appropriate throughout this age range.

We found that both height and weight independently predicted FRC in our cohort. This contrasts with data from Lum et al. [17], who found that height, age and sex were independent predictors of FRC in their cohort. It should be noted, however, that the age range of their population extended from infancy to adolescence. During the period from early childhood to adolescence, pubertal changes can result in higher variability in weight with increasing height. Therefore, pubertal changes during this period likely influenced the association between FRC and weight, which appears to be independent of sex. Only seven out of 180 subjects in our study were classified as obese (body mass index (BMI) z-scores $\geqslant 2$ ) and, therefore, it is possible that predicted FRC values could be underestimated in obese individuals.

\section{Technical and physiological factors}

In order to further understand the technical and physiological factors that may influence ventilation distribution outcome during childhood and adolescence, we investigated whether equipment-related $V_{\mathrm{D}}$ and breathing pattern influenced results. It has been reported previously that $V_{\mathrm{D}} / V_{\mathrm{T}}$ decreases with age during childhood [37] and that $V_{\mathrm{D}} / V_{\mathrm{T}}$ is positively associated with LCI outcomes [30]. We also found that $V_{\mathrm{D}} / V_{\mathrm{T}}$ was positively associated with LCI and moment ratio outcomes. Users can ensure that the effect of $V_{\mathrm{D}}$ on MBW outcomes is minimised by using the appropriate $V_{\mathrm{D}}$ reducers recommended by the manufacturer. In addition, manufacturers of MBW devices should aim to further reduce equipment-related $V_{\mathrm{D}}$ to avoid over-estimation of ventilation distribution outcomes in young children [13].

It has previously been shown that breathing pattern can influence MBW outcomes. Fixed $1 \mathrm{~L}$ breathing protocols highly influenced LCI outcomes in children compared with relaxed breathing [29]. We found that the $V_{\mathrm{T}}$ /FRC ratio was negatively associated with age and positively associated with ventilation distribution outcomes. These findings indicate that any small age dependence of ventilation distribution outcomes in our study were likely to be influenced by age dependent changes in $V_{\mathrm{D}} / V_{\mathrm{T}}$ and $V_{\mathrm{T}} / \mathrm{FRC}$ during the period from early childhood to adolescence.

\section{Strengths and limitations}

This study comprises the largest sample of healthy Caucasian children collected using a commercially available MBW device. All four centres have extensive experience in MBW testing and collected MBW data using the same equipment and measurement protocol. In addition, all the measurements were quality controlled and analysed using the same software version, equipment $V_{\mathrm{D}}$ and system settings. However, the sample size is still relatively small for the generation of reference values. In addition, the quality control has been performed by a single operator, which could have produced a bias in the study. We did not report phase III slope indices as these estimates require additional breath-by-breath quality control, which is not yet standardised. Furthermore, we did not include preschool children in this cohort for several reasons, including the limited number of preschool visits available in our data set, lack of preschool MBW standardisation at the time of measurement and differences in interfaces used for testing at this age (i.e. facemask versus mouthpiece).

\section{Recommendations for the use of reference values}

MBW outcomes are considered to be device and tracer gas specific [18-20,38] and these reference values have been generated from $\mathrm{N}_{2} \mathrm{MBW}$ data collected on the Eco Medics Exhalyzer D device. It is possible that the ULN we report for LCI and moment ratios are appropriate for other MBW systems but further work is needed to address this. Our data were analysed using Spiroware version 3.2.1, which uses different signal processing algorithms compared with previous versions (as described in the supplementary material) and which has a known influence on outcomes [21,38]. Ideally, the most robust approach for 
Spiroware users would be to reload their raw data (A-files) collected in previous software versions into the new software version in order to re-analyse them. If this is not feasible, the users should recruit age-matched healthy controls at their own centre. In addition, as we only included subjects of Caucasian origin and it is unclear whether MBW indices differ with ethnicity at this age range [17, 39], we cannot generalise these data to other ethnic groups. Future studies that include children of other ethnicities are needed.

\section{Conclusion}

This study provides reference values for $\mathrm{N}_{2} \mathrm{MBW}$ outcomes in Caucasian school aged children measured on the commercially available Eco Medics AG ultrasonic flowmeter device. Definition of the ULN over a wide age range will allow appropriate interpretation of MBW outcomes in the paediatric clinical setting.

Acknowledgements: The authors appreciate the contributions of Sandra Lüscher, Monika Graf, Linda Beul, Gisela Wirz, Sharon Krattinger, Nadja Kranz, Marisa Guidi and Insa Korten (Division of Respiratory Medicine, Dept of Pediatrics, Inselspital and University of Bern, Bern, Switzerland), of Cornelia Joachim (Division of Pediatric Pulmonology and Allergy and Cystic Fibrosis Center, Dept of Pediatrics, University of Heidelberg, Heidelberg, Germany) and of Caroline McGirr, Jasmine Grdosic and Chloe Batchelor (Telethon Kids Institute, Subiaco, Australia) to data collection. They also would like to thank the children and their families that participated in the study.

Conflict of interest: P. Anagnostopoulou has nothing to disclose. P. Latzin reports personal fees from Gilead, Novartis, Polyphor, Santhera, Schwabe, Vertex, Vifor and Zambon, as well as grants from Vertex, outside the submitted work. R. Jensen has nothing to disclose. M. Stahl reports personal fees from Vertex Pharmaceuticals, outside the submitted work. A. Harper has nothing to disclose. S. Yammine has nothing to disclose. J. Usemann has nothing to disclose. R.E. Foong has nothing to disclose. B. Spycher has nothing to disclose. G.L. Hall reports grants from the NHMRC and the Australia and USA CF Foundation, and non-financial support (loan of equipment) from ndd, during the conduct of the study. F. Singer reports personal fees from Vertex and Novartis, outside the submitted work. S. Stanojevic has nothing to disclose. M.A. Mall reports grants from the German Federal Ministry of Education and Research (82DZL004A1) and the Einstein Foundation Berlin (EP-2017-393), during the conduct of the study, and personal fees advisory board work, consultancy and lectures from Boehringer Ingelheim and Vertex Pharmaceuticals, outside the submitted work. F. Ratjen reports grants and personal fees for consultancy from Vertex, plus personal fees for consultancy from Novartis, Bayer, Roche and Genetech, outside the submitted work. K.A. Ramsey has nothing to disclose.

Support statement: This work was funded by the Fondation Botnar, the Swiss National Foundation (grant 324730_144280/1), the Swiss Cystic Fibrosis Society (CHCF) NHMRC (AP1020555), the Cystic Fibrosis Foundation, and the Cystic Fibrosis Foundation Therapeutics (HALL14A0), the German Ministry for Education and Research (82DZL00401, 82DZL004A1 to M.A. Mall), and the German Cystic Fibrosis Association Mukoviszidose e.V. (grant 15/01 to M. Stahl). K.A. Ramsey is supported by a grant from the Swiss National Science Foundation (18-061). M.A. Mall is supported by a grant from the Einstein Foundation Berlin (EP-2017-393). The funders had no role in the study design, data collection and analysis, decision to publish, or preparation of the manuscript. Funding information for this article has been deposited with the Crossref Funder Registry.

\section{References}

1 Gustafsson PM, Aurora P, Lindblad A. Evaluation of ventilation maldistribution as an early indicator of lung disease in children with cystic fibrosis. Eur Respir J 2003; 22: 972-979.

2 Ramsey KA, Rosenow T, Turkovic L, et al. Lung clearance index and structural lung disease on computed tomography in early cystic fibrosis. Am J Respir Crit Care Med 2016; 193: 60-67.

3 Aurora P, Gustafsson P, Bush A, et al. Multiple breath inert gas washout as a measure of ventilation distribution in children with cystic fibrosis. Thorax 2004; 59: 1068-1073.

4 Singer F, Abbas C, Yammine S, et al. Abnormal small airways function in children with mild asthma. Chest 2014; 145: 492-499.

5 Boon M, Vermeulen FL, Gysemans W, et al. Lung structure-function correlation in patients with primary ciliary dyskinesia. Thorax 2015; 70: 339-345.

6 Stanojevic S, Davis SD, Retsch-Bogart G, et al. Progression of lung disease in preschool patients with cystic fibrosis. Am J Respir Crit Care Med 2017; 195: 1216-1225.

7 O'Neill K, Tunney MM, Johnston E, et al. Lung clearance index in adults and children with cystic fibrosis. Chest 2016; 150: 1323-1332.

8 Stahl M, Wielputz MO, Graeber SY, et al. Comparison of lung clearance index and magnetic resonance imaging for assessment of lung disease in children with cystic fibrosis. Am J Respir Crit Care Med 2017; 195: 349-359.

9 Kent L, Reix P, Innes JA, et al. Lung clearance index: evidence for use in clinical trials in cystic fibrosis. J Cyst Fibros 2014; 13: 123-138.

10 Amin R, Subbarao P, Jabar A, et al. Hypertonic saline improves the LCI in paediatric patients with CF with normal lung function. Thorax 2010; 65: 379-383.

11 Ratjen F, Hug C, Marigowda G, et al. Efficacy and safety of lumacaftor and ivacaftor in patients aged 6-11 years with cystic fibrosis homozygous for F508del-CFTR: a randomised, placebo-controlled phase 3 trial. Lancet Respir Med 2017; 5: 557-567.

12 Singer F, Kieninger E, Abbas C, et al. Practicability of nitrogen multiple-breath washout measurements in a pediatric cystic fibrosis outpatient setting. Pediatr Pulmonol 2013; 48: 739-746.

13 Robinson PD, Latzin P, Verbanck S, et al. Consensus statement for inert gas washout measurement using multiple- and single-breath tests. Eur Respir J 2013; 41: 507-522.

14 Yammine S, Singer F, Abbas C, et al. Multiple-breath washout measurements can be significantly shortened in children. Thorax 2013; 68: 586-587. 
15 Stanojevic S, Jensen R, Sundaralingam D, et al. Alternative outcomes for the multiple breath washout in children with CF. J Cyst Fibros 2015; 14: 490-496.

16 Fuchs SI, Eder J, Ellemunter $\mathrm{H}$, et al. Lung clearance index: normal values, repeatability, and reproducibility in healthy children and adolescents. Pediatr Pulmonol 2009; 44: 1180-1185.

17 Lum S, Stocks J, Stanojevic S, et al. Age and height dependence of lung clearance index and functional residual capacity. Eur Respir J 2013; 41: 1371-1377.

18 Jensen R, Stanojevic S, Gibney K, et al. Multiple breath nitrogen washout: a feasible alternative to mass spectrometry. PLoS One 2013; 8: e56868.

19 Poncin W, Singer F, Aubriot AS, et al. Agreement between multiple-breath nitrogen washout systems in children and adults. J Cyst Fibros 2017; 16: 258-266.

20 Yammine S, Lenherr N, Nyilas $\mathrm{S}$, et al. Using the same cut-off for sulfur hexafluoride and nitrogen multiple-breath washout may not be appropriate. J Appl Physiol 2015; 119: 1510-1512.

21 Summermatter S, Singer F, Latzin P, et al. Impact of software settings on multiple-breath washout outcomes. PLoS One 2015; 10: e0132250.

22 Anagnostopoulou P, Yammine S, Schmidt A, et al. False normal lung clearance index in infants with cystic fibrosis due to software algorithms. Pediatr Pulmonol 2015; 50: 970-977.

23 Subbarao P, Milla C, Aurora P, et al. Multiple-breath washout as a lung function test in cystic fibrosis. A Cystic Fibrosis Foundation workshop report. Ann Am Thorac Soc 2015; 12: 932-939.

24 Houltz B, Green K, Lindblad A, et al. Tidal $\mathrm{N}_{2}$ washout ventilation inhomogeneity indices in a reference population aged 7-70 years. Eur Respir J 2012; 40: Suppl. 56, P3797.

25 Verbanck S, Van Muylem A, Schuermans D, et al. Transfer factor, lung volumes, resistance and ventilation distribution in healthy adults. Eur Respir J 2016; 47: 166-176.

26 Stanojevic S, Wade A, Stocks J, et al. Reference ranges for spirometry across all ages: a new approach. Am J Respir Crit Care Med 2008; 177: 253-260.

27 Yammine S, Nyilas S, Casaulta C, et al. Function and ventilation of large and small airways in children and adolescents with inflammatory bowel disease. Inflamm Bowel Dis 2016; 22: 1915-1922.

28 Ramsey KA, Foong RE, Grdosic J, et al. Multiple-breath washout outcomes are sensitive to inflammation and infection in children with cystic fibrosis. Ann Am Thorac Soc 2017; 14: 1436-1442.

29 Yammine S, Singer F, Gustafsson P, et al. Impact of different breathing protocols on multiple-breath washout outcomes in children. J Cyst Fibros 2014; 13: 190-197.

30 Benseler A, Stanojevic S, Jensen R, et al. Effect of equipment dead space on multiple breath washout measures. Respirology 2015; 20: 459-466.

31 Jensen R, Stanojevic S, Klingel M, et al. A systematic approach to multiple breath nitrogen washout test quality. PLoS One 2016; 11: e0157523.

32 Centers for Disease Control and Prevention, National Center for Health Statistics. CDC growth charts: United States. www.cdc.gov/growthcharts/ Date last accessed: February 19, 2017. Date last updated: May 30, 2000.

33 Gustafsson PM, Bengtsson L, Lindblad A, et al. The effect of inert gas choice on multiple breath washout in healthy infants: differences in lung function outcomes and breathing pattern. J Appl Physiol 2017; 123: 1545-1554.

34 Stahl M, Joachim C, Wielputz MO, et al. Comparison of lung clearance index determined by washout of $\mathrm{N}_{2}$ and $\mathrm{SF}_{6}$ in infants and preschool children with cystic fibrosis. J Cyst Fibros 2019; 18: 399-406.

35 Raaijmakers L, Jensen R, Stanojevic S, et al. Validation of multiple breath washout devices. J Cyst Fibros 2017; 16: e22-e23.

36 Verbanck S, Paiva M, Schuermans D, et al. Relationships between the lung clearance index and conductive and acinar ventilation heterogeneity. J Appl Physiol 2012; 112: 782-790.

37 Robinson PD, Latzin P, Ramsey KA, et al. Preschool multiple-breath washout testing. An official American Thoracic Society technical statement. Am J Respir Crit Care Med 2018; 197: e1-e19.

38 Kentgens AC, Guidi M, Korten I, et al. Infant multiple breath washout using a new commercially available device: ready to replace the previous setup? Pediatr Pulmonol 2018; 53: 628-635.

39 Sonnappa S, Bastardo CM, Stafler P, et al. Ethnic differences in fraction of exhaled nitric oxide and lung function in healthy young children. Chest 2011; 140: 1325-1331. 